\title{
Ultrasound of Acute Appendicitis in the Admission Room of a Multidisciplinary Surgical Hospital
}

\author{
Mariya A.Vasilyeva, MD, $\mathrm{PhD}^{1,2}$; Aleksandr Yu. Vasilyev, MD, $\mathrm{ScD}^{1^{*}}$; Dmitry A. Lezhnev, \\ $\mathrm{MD}, \mathrm{ScD}^{1}$; Elena A. Egorova, MD, $\mathrm{ScD}^{1}$; Margarita V. Smyslenova, $\mathrm{MD}, \mathrm{ScD}^{1}$; \\ Viktor P. Truten, MD, $\mathrm{ScD}^{1}$; Viktoriya V. Petrovskaya, $\mathrm{MD}, \mathrm{ScD}^{1}$; \\ Polina E. Molchanova, $\mathrm{MD}^{2}$; Margarita O. Dutova, $\mathrm{MD}^{1}$ \\ ${ }^{1}$ Moscow State University of Medicine and Dentistry named after A. I. Evdokimov \\ ${ }^{2}$ Municipal Clinical Hospital named after S. I. Spasokukotsky \\ Moscow, the Russian Federation
}

\begin{abstract}
Ultrasound (US) for diagnosing acute appendicitis and its complications in the admission room may be used as a primary diagnostic method in urgent patients. A total of 180 adult patients underwent US for suspected appendicitis; these results showed high possibilities in pathology screening and differential diagnosis from similar clinical diseases. The accuracy of US in detecting acute appendicitis with obvious clinical findings and typical position is $100 \%$, but in retrocecal and retroperitoneal forms, it decreases to $84 \%$. With our algorithm of the right iliac and localized pain regions, US scanning optimizes the screening of patients with suspected AA presented in the admission room. (International Journal of Biomedicine. 2018;8(4):324-326.)
\end{abstract}

Key Words: appendix $\bullet$ acute appendicitis $\bullet$ ultrasound $\bullet$ diagnostic algorithm

\section{Introduction}

The optimization of the diagnostic algorithm of acute appendicitis (AA) remains a serious problem. This is the most frequent pathology in urgent surgery: it amounts to $80 \%-85 \%$ of emergency procedures. The prevalence of this disease is estimated to be 5 persons per 1000 annually. ${ }^{(1,2)}$ Misdiagnosing appendicitis leads to unnecessary surgery and excision of unaltered appendices in about $15 \%$ of cases, despite the obvious clinical manifestations. ${ }^{(1,3)}$ This raises the necessity of imaging methods that could increase the diagnostic accuracy of the appendiceal destruction stage in the admission room, differentiate it from other diseases, select the surgical approach, avoid harming a patient with an unnecessary intervention and reduce the diagnostic time.

Since 1980, ultrasonography has been a useful method to visualize the altered appendix, and in 1986, it became the main accessory modality with an adjacent compression technique during abdominal examination. Many surgeons emphasize the

"Corresponding author: Prof. Alexander Yu. Vasilyev, MD, ScD. Department of Radiology, Moscow State University of Medicine and Dentistry n. a. A. I. Evdokimov, Moscow, Russia.E-mail:auv62@mail.ru importance of imaging methods only in situations where no more exact clinical symptoms exist. If the clinical presentation and the anamnesis are clear, the diagnosis of AA is established without extra diagnostic methods. ${ }^{(1,2,4,5)}$

According to a number of authors, the US accuracy for this kind of pathology varies from $66 \%$ to $100 \%$. $^{(2,3,6)}$ The disadvantages of US are operator-dependence and the factors that complicate the visualization of the appendix, such as obesity, hyperpneumatisation of bowel or inadequate transducer compression. There is a need for a scanner of expert class at the stage of the admission room of a multidisciplinary hospital because the technical data are extremely important in abdominal ultrasound examination. ${ }^{(1,2,7)}$ Moreover, the differentiation of AA types and their complication facilitates the choice of surgical approach and patient treatment.

The purpose of our study was to estimate the possibilities and the diagnostic efficacy of US examination of patients with suspected AA in the admission room.

\section{Materials and Methods}

We analyzed the sonographic data of 180 patients between 18 and 78 years of age (mean age, $25 \pm 4$ years) 
who presented to the admission room of Municipal Clinical Hospital, named after S.I. Spasokukotsky, from September 2017 to September 2018 with suspected AA. The clinical symptoms of this disease and its sonographic signs were considered. The clinical examination in a surgeon's cabinet at the admission room was in the first stage; the second one included the US examination of abdominal, retroperitoneal, and pelvic areas focused on the right side of iliac region. Ultrasound was performed with a Sonoscape S20 (SonoScape, China) with different types of modes and probes according to the patient's physique, mass of subcutaneous tissue, intestinal pneumatosis and imaging quality. If the US findings of AA were absent, we used abdominal computed tomography (CT) in the surgical department. All findings were confirmed by surgical intervention or $\mathrm{CT}$ if there were no signs of AA. We accord high priority to the diagnostic accuracy at the stage of the admission room as the most important place for medical sorting in the multidisciplinary hospital.

\section{Results}

From 180 patients who presented to the admission room with suspected AA, the US examination confirmed this diagnosis in $122(68 \%)$ cases. It was impossible to form a diagnosis with US in $18(10 \%)$ patients due to an atypical appendix position and some constitutional features, but in 16 cases out of the 122, US showed inflammation symptoms, such as paracolic consolidation, bowel wall thickening in the right side of the iliac region, and peritoneal effusion confirmed on $\mathrm{CT}$. The presence of an unaltered appendix or other pelvic and retroperitoneal pathology (also confirmed on CT) refuted the diagnosis of AA in 40 (22\%) patients; 4 (10\%) patients from this group were found to have an unaltered appendix in the typical position.

One hundred and forty patients with a confirmed AA had different appendix positions (Table 1):

- Typical $[105(75 \%)]$ - the inflammatory changes were detected on US in $100 \%$

- Retrocecal and retroperitoneal [19 (14\%)] - in 11 (58\%) patients the diagnosis was confirmed on US, $8(42 \%)$ patients underwent $\mathrm{CT}$ due to absence of clear features of AA on US

- Ascending [7 (5\%)] - detected on US in $6(86 \%)$ patients

- Medial [9 (6\%)] position could not be visualized on US because of atypical position and unrepresentative clinical symptoms (differential diagnosis included acute pancreatitis and mesenterial thrombosis)

\section{Table 1.}

Diagnostic accuracy of $U S$ in detecting $A A$ at the admission room stage

\begin{tabular}{|l|c|c|c|c|}
\hline \multirow{2}{*}{\multicolumn{1}{|c|}{ Appendix positions }} & \multicolumn{2}{c|}{ Found } & \multicolumn{2}{c|}{ Not found } \\
\cline { 2 - 5 } & Total & $\%$ & Total & $\%$ \\
\hline Typical & 105 & 100 & 0 & 0 \\
\hline Retrocecal and retroperitoneal & 11 & 58 & 8 & 42 \\
\hline Ascending & 6 & 86 & 1 & 14 \\
\hline Medial and other atypical variants & 0 & 0 & 9 & 100 \\
\hline
\end{tabular}

From a sufficient number of results, we can conclude that the efficiency of US in detecting an appendix in its typical position was $100 \%$; in retrocecal and retroperitoneal types, it was possible to visualize the appendix in over $50 \%$. It was not practical to form a diagnosis in medial and retroperitoneal positions at the stage of the admission room due to a time limit, absence of patient preparation for examination, or atypical clinical presentation mimicking another acute surgical disease (Table 2). It was found that the specificity in detecting AA by ultrasound was $100 \%$; the accuracy rate was also high - the absence of false-positive results proved this fact. We confirmed a diagnosis of AA only if the appendix was obviously visualized to reduce the diagnostic time and to avoid unnecessary examinations that could increase time to surgical intervention.

Table 2.

Diagnostic efficacy of US in detecting appendix in different positions

\begin{tabular}{|l|c|c|c|}
\hline Appendiceal positions & Sensitivity,\% & Specificity,\% & Accuracy, \% \\
\hline Typical & 100 & 100 & 100 \\
\hline $\begin{array}{l}\text { Retrocecal and } \\
\text { retroperitoneal }\end{array}$ & 58 & 100 & 88 \\
\hline Ascending & 86 & 100 & 98 \\
\hline $\begin{array}{l}\text { Medial and other } \\
\text { atypical variants }\end{array}$ & 0 & 100 & 84 \\
\hline
\end{tabular}

The US diagnostic algorithm of patients presented to the admission room with suspected AA was completed. In cases of typical clinical findings, the right iliac region should be carefully examined: firstly, we visualized the head of the caecum and the possible changes in tissue echogenicity, wall thickness, the number of layers, and the presence of effusion and infiltration. Further, we estimated the appendix: its diameter $(>6 \mathrm{~mm}-$ inflamed), wall thickness $(>1.5 \mathrm{~mm}$ - inflamed) and echogenicity, and the presence of fluid or inclusions into the appendiceal lumen (Figure 1).

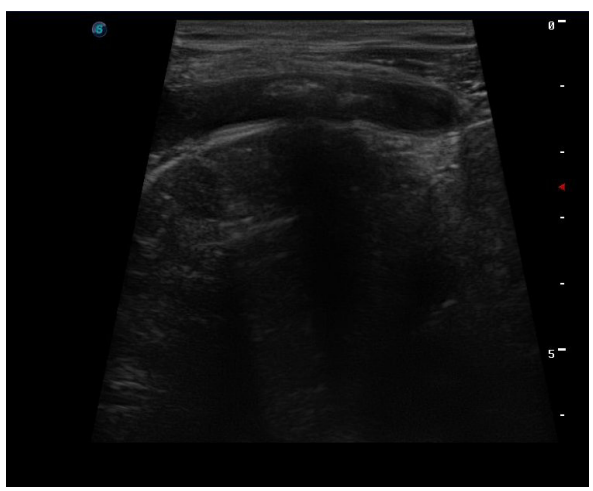

Fig. 1.

US. Inflamed appendix in typical position: diameter of $8-9 \mathrm{~mm}$, hyperechogenicity and irregular wall with thickness of $2 \mathrm{~mm}$, fluid into appendiceal lumen.

If there are some clinical findings but there is no appendix in the typical position when a patient is lying on 
his back, he should be turned on his left side to exclude the retrocecal appendiceal position (Figure 2).

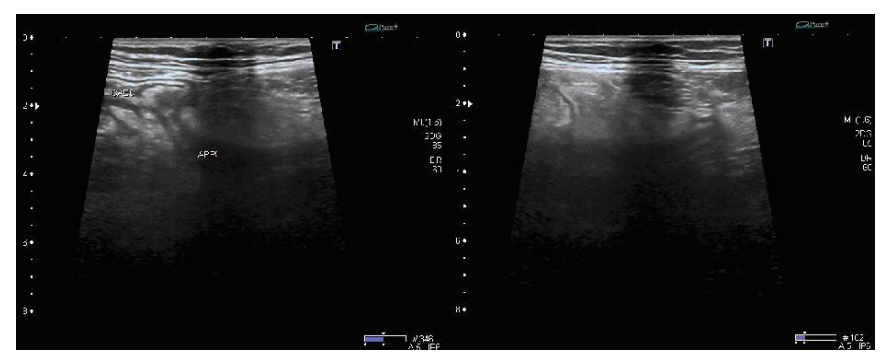

Fig. 2.

US. Inflamed appendix in retrocecal position. Appendix is located behind the bowel loop, appendiceal diameter is $8 \mathrm{~mm}$; hyperechogenic walls with thickness of $2.5 \mathrm{~mm}$. Fluid into appendiceal lumen, periappendicular infiltration. Appendiceal orifice and its origin from the cecal head are shown.

The changes of echogenicity and wall thickness of the appendix permit us to define a type of AA and its complications and, consequently, to choose a surgical approach (Figure 3 and 4).

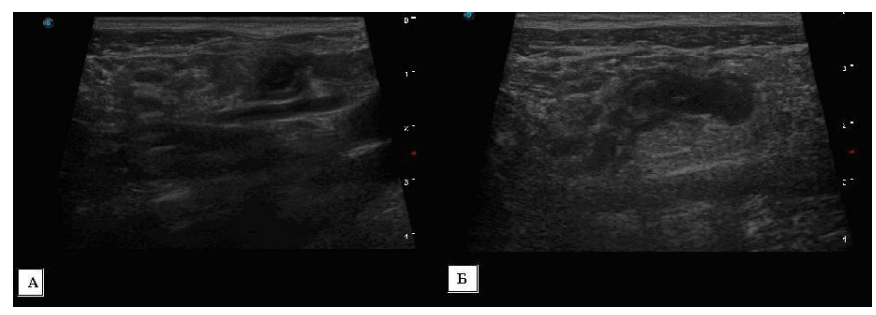

Fig. 3.

US. A-phlegmonous appendicitis: target-sign, hyperechogenicity, wall thickness of 2-2.5 mm, fluid into appendiceal lumen. B-gangrenous appendicitis: erased margins, absence of layers differentiation in middle and upper thirds of appendix without target-sign.

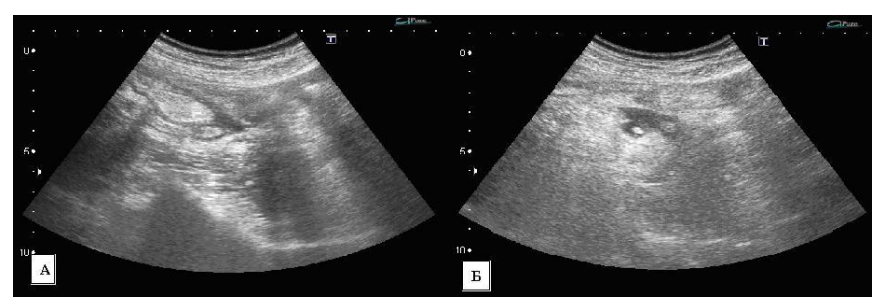

Fig. 4.

Perforation of destructive phlegmonous appendicitis with periappendicular infiltration and abscess in the apex. A-inflamed appendix with periappendicular infiltration, hyperechogenic walls with thickness of 2-2.5 $\mathrm{mm}$ and fluid collection in the apex. $B-$ heterogeneous fluid collection with irregular blurred margins and hyperechogenic inclusions (abscess).

\section{Conclusion}

Ultrasound showed a high efficacy in detecting acute appendicitis, revealed the inflammation findings and appendiceal destructive changes, and provided the differential diagnosis to clinically similar diseases. US allows us to confirm the reliable diagnosis in cases of appendiceal accessible position and exact clinical assignment, even if the patient flow is high.

- The accuracy of ultrasound in detecting appendicitis with obvious clinical findings and typical position is $100 \%$, but in retrocecal and retroperitoneal forms, it decreases to $84 \%$.

- Ultrasound is a prime modality for the mass examination of patients presented to the admission room in view of the absence of a radiation dose and high imaging quality using a US-system of expert class.

- Absence of ultrasound findings of acute appendicitis mostly confirms absence of appendiceal inflammation.

- Computed tomography should be used if the clinical presentation differs from ultrasound findings.

- Ultrasound of the right iliac and localized pain regions should be implemented into daily practice to increase the US accuracy and reduce computed tomography and laparoscopy utilization.

With our algorithm of the right iliac and localized pain regions, ultrasound scanning optimizes the screening of patients with suspected acute appendicitis presented in the admission room.

\section{Conflict of interest}

The authors declare that they have no competing interests. There was no financial support or sponsorship.

\section{References}

1. Yabunaka K, Katsuda T, Sanada S, Yatake H, Fukotomi T. Sonographic examination of the appendix in acute infectious enteritis and acute appendicitis. J Clin Ultrasound. 2008;36(2):63-6.

2. Mohamad Ghasem Hanafi MG, Afshin Shiri A. Diagnostic Accuracy of Acute Appendicitis by Ultrasound in Hospital Emergency. Jentashapir J Health Res. 2018;9(3):e82639.

3. Hussain S, Rahman A, Abbasi T, Aziz T. Diagnostic accuracy of ultrasonography in acute appendicitis. J Ayub Med Coll Abbottabad. 2014;26(1):12-7.

4. Cohen B, Bowling J, Midulla P, Shlasko E, Lester $\mathrm{N}$, Rosenberg $\mathrm{H}$, et al. The non-diagnostic ultrasound in appendicitis: is a non-visualized appendix the same as a negative study? J Pediatr Surg. 2015;50(6):923-7. doi: 10.1016/j.jpedsurg.2015.03.012.

5. Shogilev DJ, Duus N, Odom SR, Shapiro Nl. Diagnosing appendicitis: evidence-based review of the diagnostic approach in 2014. West J Emerg Med. 2014;15(7):859-71. doi: 10.5811/westjem.2014.9.21568.

6. Ly DL, Khalili K, Gray S, Atri M, Hanbidge A, Thipphavong S. When the appendix is not seen on ultrasound for right lower quadrant pain: does the interpretation of emergency department physicians correlate with diagnostic performance? Ultrasound Q. 2016;32(3):290-5. doi: 10.1097/ RUQ.0000000000000214.

7. Orscheln ES, Trout AT. Appendiceal diameter: CT versus sonographic measurements. Pediatr Radiol. 2016;46(3):31621. doi: $10.1007 / \mathrm{s} 00247-015-3491-9$. 